\title{
Margin Involved by Sarcoma
}

National Cancer Institute

\section{Source}

National Cancer Institute. Margin Involved by Sarcoma. NCI Thesaurus. Code C159233.

The margin of the sample has sarcoma. 